\title{
First Metatarsophalangeal Joint Arthrodesis: Comparison of Mesenchymal Stem Cell Allograft versus Autogenous Bone Graft Fusion Rates*
}

\author{
John Anderson ${ }^{1}$, Nathan Jeppesen ${ }^{2}$, Myron Hansen ${ }^{3}$, Chad Brady ${ }^{4}$, Adam Gough ${ }^{5}$, Zflan Fowler ${ }^{1}$ \\ ${ }^{1}$ Alamogordo Orthopaedics, Alamogordo, USA \\ ${ }^{2}$ Scripps Mercy-Kaiser Hospital, San Diego, USA \\ ${ }^{3}$ Cactus Foot and Ankle, Gilbert, USA \\ ${ }^{4}$ Presbyterian, Albuquerque, USA \\ ${ }^{5}$ Gila Regional Medical Center, Silver City, USA \\ Email: jsdsanderson@aol.com
}

Received January 23, 2013; revised February 25, 2013; accepted March 6, 2013

Copyright (c) 2013 John Anderson et al. This is an open access article distributed under the Creative Commons Attribution License, which permits unrestricted use, distribution, and reproduction in any medium, provided the original work is properly cited.

\begin{abstract}
With patients undergoing first time 1st metatarsophalangeal joint arthrodesis using graft material when it was required to fill cystic bone voids, we retrospectively compared the time to fusion (clinical and radiographic), and non-union rate between the patient's own bone autograft $(n=62)$ versus a mesenchymal stem cell impregnated allograft group $(n=51)$. A third control group $(n=52)$ was included in which an end-to-end arthrodesis was performed and no graft interposition was used or necessary. The non-union rate was $4 \%(n=2)$ in the control group, $5.9 \%(n=4)$ in the autograft group, and $9.5 \%(n=5)$ in the mesenchymal stem cell allograft group. The time for radiographic fusion was 6.46 weeks for the control group, 6.52 weeks for the autograft group, and 6.53 weeks for the mesenchymal stem cell allograft group. The difference in time to clinical and radiographic union and the non-union rate were not found to be statistically significant among all 3 groups. Patient comorbidities and their possible effects on union rates were also analyzed within the populations. Some comorbidities had statistically significantly non-unions within the population, notably smoking $(\mathrm{p}=0.024)$ and Rheumatoid arthritis $(\mathrm{p}=0.001)$, however the populations were fairly small. The use of allogeneic bone graft impregnated with mesenchymal stem cells yields a similar fusion rate as with the use of autologous bone graft harvested from the surrounding area. The allograft impregnated with mesenchymal stem cells is a viable alternative yielding similar results when local autogenous bone graft is not available, not obtainable, or conditions warrant its use.
\end{abstract}

Keywords: Metatarsophalangeal Joint; Bone Autograft; Mesenchymal Stem Cell

\section{Introduction}

Bone grafting is indicated for many surgical procedures. The 1st metatarsophalangeal joint (MPJ), in a large proportion of cases, does not need a bone graft interposition while attempting to achieve fusion. However, various situations and patient conditions may affect the quality of the bone that makes bone grafting necessary for optimum results for the patient. A consequence of some systemic diseases and end stage deformity of the 1st MPJ can be the formation of soft bone, bony voids, and bony cysts [1-3]. In situations where excessive bone resection is necessary depending on the patient condition and in

*Presented at the 2010 ACFAS abstract presentations Ft. Lauderdale FL meeting. cases where secondary to variety of intraoperative techniques, a small amount of bone graft is necessary, the surgeon must decide where best to harvest or which graft source to use. These options range from the gold standard autograft [1], to allograft, to myriad other orthobiologic materials designed to facilitate bony fusion. Many times the resected portions of the 1st metatarsal head or bone from a secondary surgical procedure may provide sufficient autologous bone quality during the 1st MPJ fusion. This may be used to facilitate bony union at these sites.

Mesenchymal stem cells (MSC) that are impregnated in cancellous allograft are becoming more commonly used to aid in achieving adequate fusion rates. MSCs are multipotential adult stem cells that can differentiate into 
bone, cartilage, or fat cells depending on local needs and environment [4]. MSCs in the arthrodesis setting will be exposed to cytokines and other local factors that will direct them in differentiation to the osteoblast cell line [5]. Donor MSCs are also advantageous in that they elicit little to no immune response in host tissue and even aid in reducing local inflammatory response [6]. The combination of MSCs with a cancellous carrier (available in several different varieties commercially) makes these grafts osteoinductive, osteoconductive, and osteogenic, theoretically closely resembling a patients autologous bone.

In the current health care age, we realize the cost and impact of increased operative time to harvest bone graft elsewhere such as the proximal tibia, the distal tibia, the calcaneus or surrounding areas. Likewise there is also an increased era of cost containment with the expense of operative materials, i.e. orthobiologics, bone allograft including demineralized bone matrix, bone allograft and MSC products. This study compares what the literature suggests is the "gold standard", [1] which is bone autograft to allogeneic bone graft impregnated with MSCs in the context of 1st MPJ arthrodesis. There are no long term outcome studies comparing these two groups of patients requiring 1st MPJ fusions. Our primary hypothesis is that there will be very comparable times to fusion and union rates among autograft and allograft with MSC groups in the setting of a 1st MPJ arthrodesis. This should help the surgeon with additional information in the comparison of the use of these materials. A secondary endpoint of this study was to evaluate the number of nonunions in patients with higher risk co-morbid conditions. We hypothesize that the presence of diabetes, obesity, rheumatoid arthritis (RA), or smoking will increase the rate of non-unions within our patient population.

\section{Patients and Methods}

The study was comprised of 204 consecutive patients before exclusions, from August 2004 to December 2008, undergoing 1st MPJ arthrodesis for end stage deformity correction from the practice of author JJA. The patients were divided into 3 treatment groups consisting of 1st MPJ fusion with MSC impregnated allogeneic graft, autologous bone graft, and end to end arthrodesis where no graft interposition was used or necessary. The end to end arthrodesis group was used in this study as a similar but not necessarily equivalent "control" to the other groups. Data collection used for statistical analysis was patient's age, sex, radiographic time to union, clinical time to union, non-union rate, presence of comorbid conditions, and patient satisfaction compared between 3 treatment groups.

Radiographic union was determined by visualizing obvious bony bridging [7] across the arthrodesis site in a time blinded fashion by 3 independent podiatric surgeons. Clinical union was determined by 3 criteria [7]; subjective resolution of pain about the operative site, lack of clinical motion, and full return to normal shoe gear. Patients were evaluated on a weekly basis for the first two weeks, clinically and radiographically. Then, every 2 weeks, patients were evaluated until all conditions noted previously had been met. Multiple regression analysis was performed to determine $\mathrm{p}$ values in all situations where possible. Data collection regarding additional procedures that were secondary procedures to the 1st MPJ fusion was not gathered in this study.

Patient satisfaction was determined via office visit survey. In this general survey, patients were questioned about their satisfaction with the procedure and willing-ness to have the procedure again. Patients were also questioned about their timeliness of getting back into regular shoe gear, which was 1 of the 3 criteria for clinical union determination noted above.

The following were exclusionary criteria for this study; revisional 1st MPJ fusion secondary to malunion or previous non-union, previously infected joint, history of Charcot neuroarthropathy, or history of 1st MPJ dislocation with sesamoidal fracture. A total of 39 patients were excluded based on these criteria making the total number of patients evaluated 165 .

\section{Surgical Technique}

The arthrodesis techniques were similar in each case. All procedures were done under general anesthesia with local adjunct. The primary operative procedure was a 1st MPJ fusion in each of the cases. The 1st MPJ joint was approached via dorsal medial approach and a linear dissection performed. The bone was curettaged on both sides of the joint down to the subchondral bleeding bone via rongeur, burr, and curettage technique. At that time a determination was made by the surgeon whether or not use of bone graft was necessary. It was deemed necessary in cases where there was soft bone, bone voids, or bone cysts about the fusion site (Figure 1).

If a secondary surgical procedure was done and excess bone of necessary quality was available or the dorsal medial 1st metatarsal bone had enough cancellous quality bone to use as a graft then this graft was used. If an adjunct procedure was performed and other graft elsewhere such as phalanx or metatarsal bone was available, this bone was used. The autogenous graft was reduced to cancellous bone chips in all cases, as they were not intended for structural support.

If there were no secondary procedures performed from which bone could be obtained or the bone obtained from these areas was not of adequate quality then allogeneic impregnated MSC graft material was used. The graft was only used as a bone void filler or adjunct for soft bone, 
but was not used as a strut or meant for mechanical sup-port of the fusion or fixation. Multiple screw fixations, 2 - 5 crossed screws, were used for all cases. The appropriate number of screws used was determined by the surgeon until it was felt the 1st MPJ arthrodesis site was adequately fixated (Figure 2).

\section{Results}

Tables 1 to 4 depict the statistical results of the study. The patients in this study were well distributed between the treatment groups with regard to gender and age as

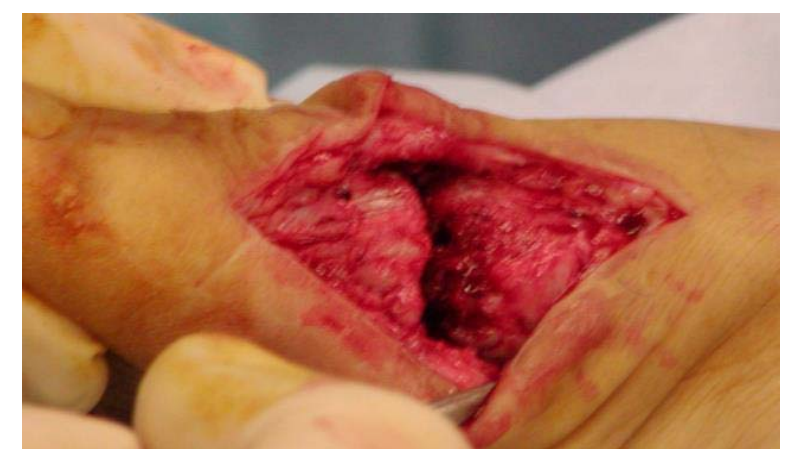

Figure 1. Prepared joint surfaces with cystic and osteopenic changes to the 1st metatarsal head.

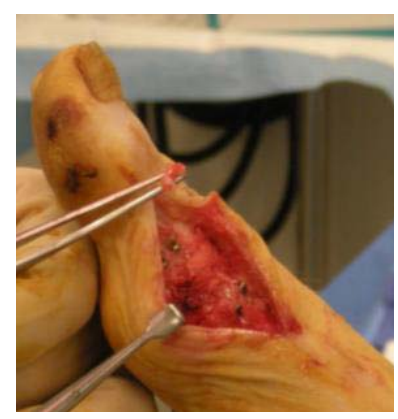

(a)

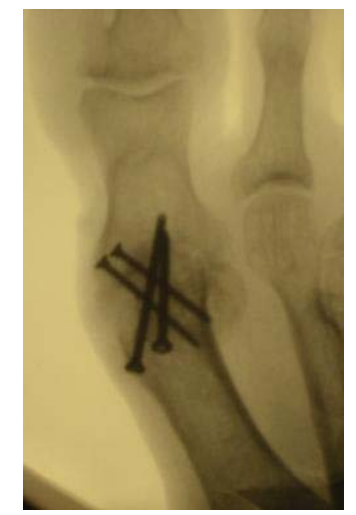

(b)

Figure 2. (a) Intra-operative and fluoroscopic view of the 1st MPJ fusion fixation technique used; (b) 2 - 5 crossed screws were used until the 1st MPJ was felt to be adequately fixated. depicted in Tables 1 and 2.

The average radiographic time for fusion in the endto-end arthrodesis was 6.76 weeks (range from 4 to 12). The time for radiographic fusion in the autograft group was 6.69 weeks (range 4 -11). The average radiographic time for fusion in the allograft MSC group was 7.0 weeks (range 4 to 12). An ANOVA test was used and revealed a p value of 0.676 which showed no clinically statistical significance between the treatment groups that did achieve fusion. However this data does not take into consideration the non-unions as a time period for union could not be calculated for these patients (Table 2).

The average clinical and functional time for fusion in the end-to-end arthrodesis was 6.46 weeks (range from 4 to 10). The time for clinical fusion in the autograft group was 6.52 weeks (range 4 -11). The average time to clinical fusion in the allograft MSC group was 6.53 weeks (range 4 to 12). A Scheffe test of homogenous subsets was used and revealed a p value of 0.966 which showed no clinical statistical significance between the treatment groups. All patients in this study achieved clinical or functional union regardless of radiographic presence of non-union (Table 2).

There were a total of 5 non-unions in the MSC allograft group, 4 non-unions in the autograft group, and 2 non-unions in the control group. A Pearson chi square was used to determine that the $\mathrm{p}$ value between all 3 treatment groups was 0.209 . A 2 sample test of proportions was used to more precisely calculate the $p$ value of the non-union rates between only the autograft group and the MSC allograft group, which was 0.177 (Table 2).

The percentage of procedures achieving fusion in the control group was $96.0 \%$, in the autograft group was 94.2\%, and in the MSC allograft group was $90.5 \%$ (Table 2).

The patients that would have the procedure again and were satisfied with the procedure were $96 \%$ in the end to end fusion group, $98 \%$ in the autograft group, and $92 \%$ in the allograft/MSC group. There was no statistical significance between the 3 treatments groups (Table 3 ).

There were no non-unions in the 12 diabetics in the study. There were 2 non-unions in 5 obese patients which

Table 1. Gender distribution between groups.

\begin{tabular}{cccccc}
\hline & & \multicolumn{2}{c}{ Treatment Group } & & Total \\
\cline { 3 - 6 } & & Allograft & Autograft & End to End & \\
\hline Gender & Male & 20 & 31 & 21 & 72 \\
& Female & 31 & 31 & 31 & 93 \\
Total & & 51 & 62 & 52 & 165 \\
\hline
\end{tabular}

Pearson Chi-square $=1.650, \mathrm{p}=0.438$. 
Table 2. Comparison between the 3 treatment groups.

\begin{tabular}{|c|c|c|c|c|c|c|}
\hline & $\mathrm{N}$ & Mean Age & $\begin{array}{l}\text { Time to Clinical } \\
\text { Union (Weeks) }\end{array}$ & $\begin{array}{l}\text { Time to Radiographic } \\
\text { Union (Weeks) }\end{array}$ & $\begin{array}{l}\text { Total Radiographic } \\
\text { Non-Unions }\end{array}$ & $\begin{array}{c}\% \text { of Radiographic } \\
\text { Fusion }\end{array}$ \\
\hline $\begin{array}{l}\text { Allograft } \\
\text { MSC }\end{array}$ & 51 & $\begin{array}{c}62.2 \pm 1.98 \\
\text { (Range } 33-89 \text { ) }\end{array}$ & $\begin{array}{c}6.53 \pm 1.39 \\
\text { (Range } 4-12 \text { ) }\end{array}$ & $\begin{array}{c}7 \pm 1.98 \\
\text { (Range } 4-12 \text { ) }\end{array}$ & 5 & $90.5 \%$ \\
\hline Autograft & 62 & $\begin{array}{c}62.16 \pm 1.7 \\
\text { (Range } 40-88 \text { ) }\end{array}$ & $\begin{array}{c}6.52 \pm 1.46 \\
\text { (Range } 4-12 \text { ) }\end{array}$ & $\begin{array}{c}6.69 \pm 1.70 \\
\text { (Range } 4-11 \text { ) }\end{array}$ & 4 & $94.1 \%$ \\
\hline $\begin{array}{l}\text { End to End } \\
\text { Arthrodesis }\end{array}$ & 52 & $\begin{array}{c}61.71 \pm 1.72 \\
\text { (Range } 39-80 \text { ) }\end{array}$ & $\begin{array}{c}6.46 \pm 1.31 \\
\text { (Range } 4-10 \text { ) }\end{array}$ & $\begin{array}{c}6.76 \pm 1.72 \\
\text { (Range } 4-12 \text { ) }\end{array}$ & 2 & $96.0 \%$ \\
\hline $\mathrm{p}$ value & & $0.967^{\mathrm{a}}$ & $0.966^{\mathrm{b}}$ & $0.676^{\mathrm{a}}$ & $\begin{array}{c}0.209^{\mathrm{c}}, 0.177^{\mathrm{d}} \\
0.296^{\mathrm{e}}\end{array}$ & $\mathrm{n} / \mathrm{a}$ \\
\hline
\end{tabular}

${ }^{\mathrm{a}}$ ANOVA test; ${ }^{\mathrm{b}}$ Scheffe test of homogenous subsets; ${ }^{\mathrm{c}}$ Pearson Chi-Square; ${ }^{\mathrm{d}} 2$ sample test of proportions between only Allograft MSC and Autograft groups; ${ }^{\mathrm{E}}$ Fischer's exact test between only Allograft MSC and Autograft groups.

was statistically significant with a $\mathrm{p}$ value of 0.036 . There were 5 non-unions in the RA group of 15 patients which was statistically significant with a p value of 0.001 . There were 5 non-unions in the group of 32 patients that smoked, which was statistically significant with a p value of 0.024 . There was an overlap between the patients with obesity, RA, and that were smokers (Table 4).

\section{Discussion}

The control group that was used in this study did have a slightly different patient population than those in the autograft group and the MSC allograft group. The patients in the end to end arthrodesis groups all had sufficient bone quality with no evidence of soft bone, bonyvoids, or large bony cysts. As such these patients we expect to have a higher reported fusion rate as evidenced in the literature $[8,9]$. The patients in the control vary from

Table 3. Patient satisfaction results.

\begin{tabular}{cccccc}
\hline & \multicolumn{2}{c}{ Treatment Group } & \multicolumn{2}{r}{ Total } \\
\hline & Allograft & Autograft & End to End \\
\hline $\begin{array}{c}\text { Have Procedure } \\
\text { Again? }\end{array}$ & Yes & 47 & 61 & 50 & 158 \\
$\begin{array}{c}\text { Patient } \\
\text { Satisfaction \% } \\
\text { Total }\end{array}$ & 4 & 1 & 2 & 7 \\
\hline
\end{tabular}

Pearson Chi-square $=2.703, \mathrm{p}=0.259$.

Table 4. Co-morbid conditions and non-union rate.

\begin{tabular}{cccc}
\hline Co-Morbidity & $\mathrm{N}$ & Non-Union & $\mathrm{p}$ Value \\
\hline Diabetes & 12 & 0 & $1.00^{\mathrm{a}}$ \\
Obesity & 5 & 2 & $0.036^{\mathrm{a}}$ \\
Rheumatoid Arthritis & 15 & 5 & $0.001^{\mathrm{b}}$ \\
Smoker & 32 & 5 & $0.024^{\mathrm{b}}$ \\
\hline
\end{tabular}

${ }^{\mathrm{a}}$ Fisher's Exact test; ${ }^{\mathrm{b}}$ Pearson Chi square. the other two groups studied in that these two graft groups did have different and less than desirable bone quality. However, the autograft group and the allograft group had the same indications for the need of grafting material, and they can be compared together and appropriate results deduced. Thus, the control group is used as a guide to compare to other published data and to then serve as a control for comparing the other 2 studied groups presented here.

This study confirms our original hypothesis that autograft from surrounding areas and allogeneic bone graft impregnated with MSC have comparable fusion times.

Both are viable options for the foot and ankle surgeon to use when a bone void deficit or surgical anatomy require use of bone adjunct to help facilitate union, being evidenced from our results that the time to radiographic and clinical fusion rate and patient satisfaction are similar for each group. The non-union rate showed a trend that that the autograft group was more effective than the MSC allograft group but all parameters showed no statistically significant difference in outcome. This was most likely due to the lower total number of non-unions present among all treatment groups.

Once again it must be reiterated that these grafts were not used as structural support to obtain length in a revisional surgery. All cases were primary 1st MPJ fusions. The data presented here cannot be directly extrapolated and applied to revisional or primary cases where bone graft is used as a structural graft. Additional studies that specifically studied that premise would be necessary to make certain conclusions.

If indeed localized bone autograft can be used, then this study would support the use of that surrounding bone. However, it is realized that often time the quality and amount of bone tissue are not readily available intra-operatively. Many times there are unforeseen cystic changes, deficits, and bone voids that are realized upon denuding the cartilage down to the subchondral bone upon preparation of the 1st MPJ. We do recognize that having to harvest local graft elsewhere would increase 
the amount of operative time [10] and potentially risk donor site morbidity $[10,11]$ such as prolonged pain and infection. Our results demonstrate that the potential to eliminate these complications with the use of the MSC allograft is now a viable option for the surgeon. We feel that further study regarding cost and benefit vs. morbidity of bone graft harvesting is warranted as this was not one of the aims of this study. However, this is an appropriate question amid the era of cost containment within the current healthcare setting.

Our results also confirm previously held knowledge about the increase rate of non-union in smoking patients. As always proper patient education and encouraging the patient to refrain from smoking until union is achieved is advised. Proper patient education is also encouraged in RA and obese patients as there was shown to be an increased chance of non-union rate in these groups. These patients need to be aware of the higher risk of non-union to prepare them for realistic expectations. It has been suggested in the literature that there is an increased rate of non-unions in diabetic patients [12], but this was not evident in our patient population, contrary to our original hypothesis. However, our population of diabetics in this study was small, and we cannot truly make the conclusion that they are not at increased risk for nonunions. Larger populations would need to be studied in all of the co-morbid condition groups for the most accurate verifycation of our results.

The results of this study once again confirm that if one is faced with the need to use bone grafting for any situation discussed previously, MSC impregnated allograft is a viable alternative. If one has a choice between readily available autograft from the residual 1st metatarsal head or secondary procedures then this should be used. However, if this autograft is of poor quality or unavailable, the surgeon should feel comfortable using of the MSC impregnated allograft when the need of a graft exists.

\section{Acknowledgements}

Special thanks to Donna Agan, Ed. D. for help with data analysis and Donald Green DPM.

\section{REFERENCES}

[1] P. S. Shurnas and M. J. Coughlin, "Arthritis, Postural Disorders, and Tendon disorders,” In: M. J. Coughlin, R. A. Mann and C. L. Saltzman, Eds., Surgery of the Foot and
Ankle, 8th Edition, Mosby Elsevier, Philadelphia, 2007, pp. 803-922.

[2] I. J. Alexandar, "Hallux Metatarsophalangeal Arthrodesis,” In: H. B. Kitaoka, Ed., Master Techniques in Orthopaedic Surgery: The Foot and Ankle, 2nd Edition, Lippincott Williams and Wilkins, Philadelphia, 2002, pp. 4560.

[3] L. S. Weil Jr., "First Metatarsophalangeal Arthrodesis," In: T. J. Chang, Master Techniques in Podiatric Surgery: The Foot and Ankle, Lippincott Williams and Wilkins, Philadelphia, 2005, pp. 119-128.

[4] R. A. Zambon, D. Buskirk and M. Roberts, "Osteoprogenitor Cells Preserved in the Osteocel ${ }^{\circledR}$ Tissue Product Result in Enhanced Bone Formation,” Osiris Therapeutics, Inc., Baltimore, 2005.

[5] S. Rush, "Mesenchymal Stem Cell Allografting in Foot and Ankle Surgery," Foot \& Ankle, Vol. 3, No. 3, 2010, pp. 140-147. doi:10.1177/1938640010369638

[6] L. Walker, A. Danilkovitch, M. L. Williams, M. Lorenz, M. R. Zindrick and R. J. Linovitz, "Trinity ${ }^{\mathrm{TM}}$ Multipotential Cellular Bone Matrix Technical Monograph,” Blackstone Medical, Inc., Baltimore, 2006.

[7] G. M. Weinraub, J. M. Shuberth, M. Lee, S. Rush, L. Ford, J. Neufeld and J. Yu, "Isolated Medial Incisional Approach to Subtalar and Talonavicular Arthrodesis," Journal of Foot and Ankle Surgery, Vol. 49, No. 4, 2010, pp. 326330. doi:10.1053/j.jfas.2010.04.015

[8] G. A. Hamilton, L. A. Ford and S. Patel, "First Metatarsophalangeal Joint Arthrodesis and Revision Arthrodesis," Clinics in Podiatric Medicine and Surgery, Vol. 26, No. 3, 2009, pp. 459-473. doi:10.1016/j.cpm.2009.03.010

[9] M. J. Coughlin and P. S. Shurnas, "Hallux Rigidus: Grading and Long-Term Results of Operative Treatment," The Journal of Bone \& Joint Surgery, Vol. 85, No. 11, 2003, pp. 2072-2088.

[10] J. K. DeOrio and D. C. Farber, "Morbidity Associated with Anterior Iliac Crest Bone Grafting in Foot and Ankle Surgery,” Foot \& Ankle International, Vol. 26, No. 2, 2005, pp. 147-151.

[11] D. W. Boone, "Complications of Iliac Crest Graft and Bone Grafting Alternatives in Foot and Ankle Surgery," Foot and Ankle Clinics, Vol. 8, No. 1, 2003, pp. 1-14. doi:10.1016/S1083-7515(02)00128-6

[12] J. K. Ellington, C. P. Jones, B. E. Cohen, W. H. Davis, F. Nickisch and R. B. Anderson, "Review of 107 Hallux MTP Joint Arthrodesis Using Dome-Shaped Reamers and a Stainless-Steel Dorsal Plate,” Foot and Ankle Clinics, Vol. 31, No. 5, 2010, pp. 385-390, doi:10.3113/FAI.2010.0385 